\title{
La importancia de la ética en la profesión contable
}

\section{The importance of ethics in the accounting profesión}

\section{La importancia de la ética}

\author{
Ruby Clarita Cerino Cerino. ${ }^{(1)}$
}

Gladys Hernández Romero. Dra. ${ }^{(2)}$

${ }^{1}$ Universidad Juárez Autónoma de Tabasco, Tabasco, México. Email: rubycccerino@gmail.com ORCID: https://orcid.org/0000-0003-0691-2919

${ }^{2}$ Universidad Juárez Autónoma de Tabasco, Tabasco, México. Email: doctoragladys@ hotmail.com ORCID: http://orcid.org/0000-0002-9045-2937

Contacto: rubycccerino@gmail.com

\section{Resumen}

La presente investigación posee un diseño descriptivo, no experimental, bajo un enfoque cuantitativo, ya que se aplicaron 115 encuestas a estudiantes y profesionales Contables de la ciudad de Villahermosa, en el Estado de Tabasco, México, durante el periodo octubre 2020-marzo 2021. El objetivo general es compilar, analizar y dar a conocer la importancia de la ética en la profesión Contable del área geográfica mencionada. Los resultados señalan que la ética es de vital importancia en la vida laboral de los estudiantes y profesionales Contables, por lo que, evitan realizar prácticas que perjudiquen a la profesión y con ello a terceros, ya que éstas traen consigo consecuencias perjudiciales para el contribuyente. A su vez, la percepción que tienen los encuestados sobre la ética es como un factor imprescindible y relevante, es decir, debe estar presente en cada una de las actividades que se realizan para garantizar la calidad de la información y con ella la adecuada toma de decisiones, respecto la ética profesional como parte de los programas curriculares, es indispensable que esta sea parte de los programas de manera general, para que, desde antes de comenzar a laborar, los futuros profesionales Contables estén correctamente informados acerca de las buenas prácticas.

Palabras clave: Ética profesional, contabilidad, profesional contable, información financiera 


\begin{abstract}
The present research has a descriptive, non-experimental design, under a quantitative approach, since 115 surveys were applied to students and accounting professionals in the city of Villahermosa, in the State of Tabasco, Mexico, during the period October 2020-March 2021. The general objective is to compile, analyze and make known the importance of ethics in the accounting profession in the mentioned geographical area. The results indicate that ethics is of vital importance in the working life of students and professional accountants, so that they avoid practices that harm the profession and thus third parties, since they bring with them harmful consequences for the taxpayer. In turn, the perception that the respondents have about ethics is as an essential and relevant factor, that is, it must be present in each of the activities carried out to ensure the quality of information and with it the proper decision making, regarding professional ethics as part of the curricular programs, it is essential that this is part of the programs in general, so that, before starting to work, future accounting professionals are properly informed about good practices.
\end{abstract}

Keywords: Professional ethics, accounting, accounting professional, financial reporting

\title{
Introducción
}

En México, las empresas deben cumplir con ciertas obligaciones como contribuyentes, las cuales están establecidas en la Ley del Impuesto Sobre la Renta, en el Código Fiscal Federal y su Reglamento, una de las obligaciones principales es el llevar la Contabilidad de dichas empresas, ya sea el caso de personas morales, personas físicas con actividad empresarial y profesional, $\mathrm{u}$ otro tipo de contribuyentes mencionados en dicha Ley y Código.

Por consiguiente, la contabilidad debe ser realizada por un Licenciado en Contaduría Pública, es decir, el rol de éste para las empresas es sustancial, y debido a esto es primordial dar conocer la importancia de la ética en esta profesión.

Por lo tanto, la presente investigación se realizó con el objetivo general de compilar, analizar y dar a conocer la importancia de la ética en la profesión Contable de la ciudad de Villahermosa, estado de Tabasco, México, de manera específica, se buscó reconocer el nivel de importancia que tiene cada principio del Código de ética profesional en el desempeño laboral de los Profesionales Contables, de igual manera, identificar los principales medios para obtener conocimientos sobre dicho Código de ética, y a su vez, exponer la frecuencia en que se presentan situaciones relacionadas a la ética dentro del desempeño profesional. 
La investigación se enfocó en esta profesión debido a su importancia, ya que es pilar fundamental en el cumplimiento de las obligaciones de las empresas como contribuyentes, en el caso de las Personas Morales, la Ley del Impuesto Sobre la Renta establece, en su título II: De las personas morales, capítulo IX: De las obligaciones de las personas morales, en la fracción primera del artículo 76 lo siguiente:

Llevar la contabilidad de conformidad con el Código Fiscal de la Federación, su Reglamento y el Reglamento de esta Ley, y efectuar los registros en la misma.

De igual manera, en el caso de las Personas Físicas con actividad empresarial y profesional, la Ley del Impuesto Sobre la Renta establece, en su título IV: De las personas físicas, capítulo II: De los ingresos por actividades empresariales y profesionales, en la fracción segunda del artículo 110 lo siguiente:

Llevar contabilidad de conformidad con el Código Fiscal de la Federación y su Reglamento, tratándose de personas físicas cuyos ingresos del ejercicio de que se trate no excedan de dos millones de pesos, llevarán su contabilidad y expedirán sus comprobantes en los términos de las fracciones III y IV del artículo 112 de esta Ley.

Así como personas morales y personas físicas con actividad empresarial y profesional están obligadas a llevar la Contabilidad, de igual manera, otro tipo de contribuyentes mencionados en dicha Ley y Código se encuentran obligados o tienen la opción de cumplir con esto, de este punto deriva la importancia de la Profesión Contable en su rol dentro de las empresas

\section{¿Qué es la contabilidad?}

La contabilidad, es una técnica que permite plasmar la información financiera a través de estados financieros, para su análisis e interpretación, lo que conlleva a formarse un criterio acerca de la situación financiera de la empresa para poder tomar decisiones en favor a ésta, y tal como se planteó anteriormente, es pilar fundamental en el cumplimiento de las obligaciones de las empresas como contribuyentes, ya sea como personas morales, personas físicas con actividad empresarial y profesional, y de igual manera, como otro tipo de contribuyentes mencionados en el marco regulativo mexicano, tal como la Ley del Impuesto Sobre la Renta, el Código Fiscal de la Federación, entre otros, es por ello que la contabilidad es de vital importancia para las empresas, y por ende, el papel del Contador Público dentro de éstas, de igual manera, es de suma importancia.

De acuerdo a la NIF A-1, párrafo 3, "la Contabilidad es una técnica para el registro de las operaciones que afectan económicamente a una entidad y produce sistemática y estructuradamente información financiera”. Del 
mismo modo, Santos (2018) expone que "la contabilidad aporta información verificable, medible, y comparable, a través de la sistematización en la obtención, análisis, medición y exposición de datos, para explicar una realidad, posible de mejorarse" (p.153).

Mientras que Alva (2018) resume que "las actividades que las empresas realizan, en su totalidad, dependen de los recursos económicos, del dinero y de la contabilidad" (p.11), por otra parte, Cifuentes et al., (2018) mencionan que "en una empresa, la contabilidad sirve para llevar el control sobre todas las cuentas y operaciones diarias, tales como las de compra, venta, gastos, inversiones, entre otras” (p.181).

\section{Los estados financieros}

Los estados financieros son el resultado de toda la información generada por la contabilidad en un periodo determinado ya sea, bimestral, trimestral, semestral, anual, etc. Dichos estados financieros, a grandes rasgos muestran el estado en que se encuentra la empresa, y a su vez, el origen, aplicación y cambios habidos de los recursos, es decir el cómo fueron administrados, y de igual manera, las pérdidas y ganancias habidas. Desde el punto de vista de Elizalde (2019) "los estados financieros reflejan las operaciones o transacciones diarias que demuestra una empresa en sus actividades, siendo resumidas en la estructura exhibida como estado financiero" (p.219).

Es por ello que cada estado financiero tiene su propósito general. Teniendo lo anterior en consideración, cabe destacar que los estados financieros básicos son cuatro, siendo éstos los siguientes:

Estado de situación financiera: el propósito de este estado financiero es representar como tal, la situación económica-financiera en que se encuentra la empresa.

Estado de resultados: el propósito de este estado financiero es dar a conocer el beneficio o pérdida habida en la empresa a lo largo del ejercicio económico.

Estado de flujo de efectivo: el propósito de este estado financiero es resumir el origen de los flujos de efectivo que ha ingresado a la empresa y en qué se ha utilizado.

Estado de cambios en el capital contable: el propósito de este estado financiero es dar a conocer el beneficio o pérdida derivado de causas diferentes a los costos y gastos de la empresa, los cuales modifican el capital contable o patrimonio.

\section{¿Por qué es importante la información financiera?}


De acuerdo a la NIF A-1, párrafo 4:

La Información financiera que emana de la contabilidad, es información cuantitativa, expresada en unidades monetarias y descriptiva, que muestra la posición y desempeño financiero de una entidad, y cuyo objetivo esencial es el de ser útil al usuario general en la toma de decisiones económicas.

Por consiguiente, Fernández et al., (2019) menciona que "la información financiera se desarrolla con el fin de suministrar información valiosa, eficiente y de fácil interpretación que permita conocer las herramientas financieras (...) y su importancia para la toma de decisiones y el aprovechamiento de los recursos” (p.28). Es por esto, que la información financiera es de extrema importancia ya que permite evaluar el desarrollo de la entidad y tener pautas para la toma de decisiones acerca del futuro de la misma.

Dicho de otra manera, Gómez, Aristizabal y Fuentes (2017) presentan "tres dimensiones (usos) de la información financiera:

- Herramienta para la toma de decisiones;

- Herramienta de control en la organización; y,

- Fuente de información para usuarios internos y externos de la organización” (p.91).

Por lo que, para realizar el proceso de toma de decisiones se requiere de profesionales capaces de proporcionar y analizar minuciosamente la información financiera obtenida a través de los estados financieros, esto con la finalidad de cumplir con los objetivos de la organización, es por ello que los Administradores Financieros recurren, en la mayoría de las ocasiones, a los Contadores para el apoyo primordial de esta labor.

\section{El Profesional Contable}

Es el Licenciado en Contaduría Pública, quien brinda sus servicios profesionales ya sea de manera independiente o como empleado de una entidad, éste se puede desempeñar en ámbitos como contabilidad, costos, auditoria, fiscal, asesoría, etc. Su rol dentro de las empresas es de suma importancia ya que mediante la contabilidad surge la información financiera que funge como pauta primordial en la toma de decisiones de las empresas.

Por consiguiente, pueden desempeñarse en una sola área y a su vez, una gran cantidad de dichos profesionales se desempeñan en más de una, realizando actividades de suma importancia para el bienestar de las empresas. 
Tal como lo plantea Ostengo (2015) "el Profesional Contable competente tendrá funciones que lo involucren con toda actividad y proceso de la organización donde se requiera una toma de decisión” (p.10).

Importancia de la ética en el Profesional Contable

De acuerdo a García (2020) "La ética se refiere a los principios fundamentales de la moralidad donde algunas acciones se etiquetan como buenas o malas, correctas o incorrectas, éticas o poco éticas” (p.2), por lo que, la ética profesional es el concepto por el cual las personas que ejercen una profesión se rigen para no cometer o ser parte de actos ilícitos o de poca calidad moral.

En cada una de las profesiones se debe de tener un gran conocimiento de la ética que rige la misma, en el caso del Profesional Contable es estrictamente primordial ya que normalmente se trabaja con el capital ajeno de múltiples personas involucradas en una sociedad o empresa, o bien, con capital público.

Teniendo en consideración lo anterior, los Profesionales Contables tienen un deber especial en este aspecto ya que en ellos se deposita muchísima fe y confianza en sus acciones al momento de validar la información analizada o escrita en los registros que se hacen periódicamente a las diferentes entidades económicas, sean públicas o privadas.

Si se hace énfasis en el ámbito público de la profesión contable, se debe tener presente aún más el código de ética profesional por el hecho de que los recursos que se manejan o se interactúan no son de índole privado, sino que pertenecen a la nación o al Estado al cual se está laborando.

Siendo así, el Profesional Contable debe poner en práctica día con día el Código de ética que rige su profesión, ya que la ética en cada una de sus funciones y actividades garantizan la calidad y confiabilidad de su trabajo, puesto que, ésta profesión se caracteriza por ser recta y completamente apegada a las leyes y normas que la rigen, siendo éstas el marco por el cuál todas aquellas personas que pretenden ejercer la contabilidad deberán de seguir estrictamente para el correcto desarrollo de su carrera y de su labor como profesionista.

Tal como lo menciona autores como Colmenares, Da Costa y Cegarra (2014):

En el caso particular de los Contadores Públicos, más allá de mantener la ética, éste tendría que concentrarse en el desarrollo de sus valores éticos, los cuales constituyen la moral y la práctica de la carrera; por ello los estudiantes universitarios deben ser enseñados en valores, es decir, una educación que les permita formarse de manera integral para consolidar su mundo profesional (p.548). 
Por esta razón, es indispensable tener en consideración la ética profesional en cada una de las actividades a realizar, ya que el actuar de manera perniciosa afecta a la empresa para la que se labora, y a su vez, al prestigio de la profesión, ya que crea consigo un estereotipo incorrecto, de este modo, cumplir con un código de ética es importante para esta profesión, el mismo debería ser implícito en la formación profesional.

\section{Materiales y método}

El tipo de investigación es descriptiva ya que "se busca especificar las propiedades, las características y los perfiles de personas (...) o cualquier otro fenómeno que se someta a un análisis" (Hernández, Fernández, y Baptista, 2014, p.92) y, de igual manera es explicativa ya que "pretende establecer las causas de los sucesos o fenómenos que se estudian" (p.95).

La población muestra investigada comprende de 115 encuestados, entre ellos estudiantes y profesionales Contables, de la ciudad de Villahermosa, en el Estado de Tabasco, México, durante el periodo octubre 2020marzo 2021. Cabe mencionar que la muestra es no probabilística ya que "la elección de los elementos no depende de la probabilidad, sino de factores relacionados con el criterio del investigador" (Universidad Naval, 2016, p.38).

El presente trabajo se realizó bajo un enfoque cuantitativo ya que se aplicaron encuestas y se pretende brindar mayor importancia a los resultados obtenidos, de igual manera, se implementó el método deductivo.

Las técnicas aplicadas fueron documentales, es decir, la consulta de material bibliográfico de referencia y de estudio, tal como reportes de investigación empírica, artículos conceptuales o teoréticos, y revisión de libros. Y así mismo, técnica de campo, ya que se aplicaron encuestas de manera virtual a través de la plataforma "Google Forms". Del mismo modo, los instrumentos implementados son la recopilación, revisión y análisis de contenido bibliográfico, así como la aplicación de encuestas y el análisis de datos asistidos por computadora.

Por otra parte, la unidad de análisis es el análisis de encuestas aplicadas de manera virtual a través de la plataforma Google Forms a estudiantes y profesionales Contables, de la ciudad de Villahermosa, en el Estado de Tabasco, México, y de forma similar, poblaciones finitas constituidas por información documental tales como artículos científicos y libros.

\section{Resultados}

La presente investigación, como se mencionó anteriormente, se realizó con el objetivo general de compilar, analizar y dar a conocer la importancia de la ética en la profesión Contable de la ciudad de Villahermosa, estado 
de Tabasco, de manera específica, se buscó reconocer el nivel de importancia que tiene cada principio del Código de ética profesional en el desempeño laboral de los Profesionales Contables, de igual manera, identificar los principales medios para obtener conocimientos sobre dicho Código de ética, y a su vez, exponer la frecuencia en que se presentan situaciones relacionadas a la ética dentro del desempeño profesional.

Para poder tener un panorama amplio de la situación, se procedió a recopilar direcciones de correo electrónico de estudiantes de últimos semestres de la Licenciatura de Contaduría Pública de la Universidad Juárez Autónoma de Tabasco, los cuales ya laboraban, así como, Despachos Contables y Contadores independientes de la ciudad de Villahermosa, mediante directorios y bases de datos en línea, posteriormente se aplicaron encuestas a través de la plataforma Google Forms con preguntas cerradas de opción múltiple.

Para empezar, se enfocó primordialmente en dar a conocer si la población encuestada tiene conocimiento sobre el Código de ética de la Profesión Contable, obteniendo como resultado que el $89 \%$ de los profesionales contables señaló que si, tal como lo muestra la ilustración 1:
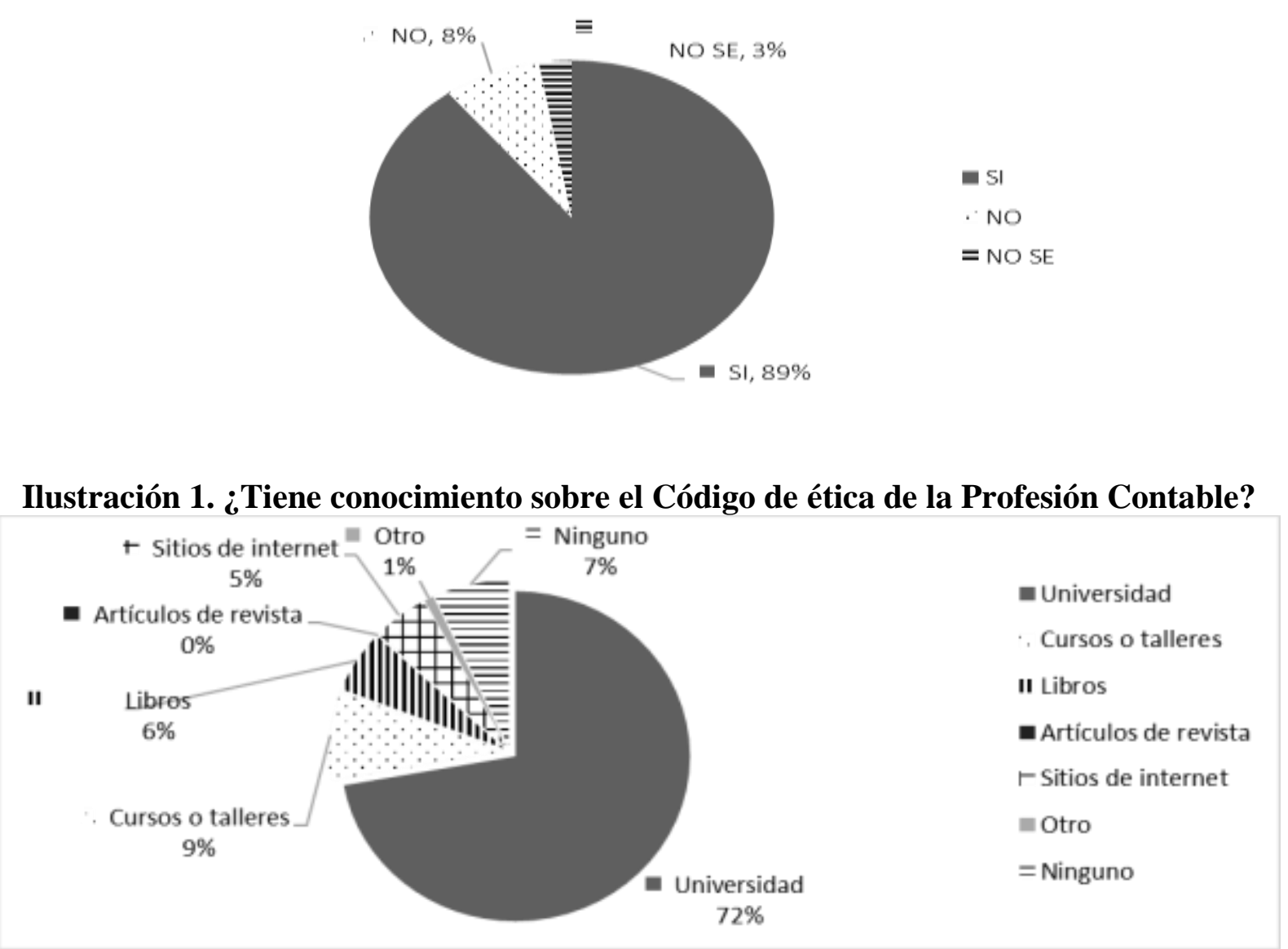


\section{Ilustración 2. ¿Mediante cual medio ha obtenido conocimiento sobre el Código de ética de la Profesión Contable?}

En consideración a lo anterior, se procedió a exponer mediante cual medio han obtenido dichos conocimientos sobre el Código de ética de la Profesión Contable, dando como resultados que el $72 \%$ de los encuestados obtuvieron dichos conocimientos gracias a la educación superior, es decir, la Universidad, siendo éstos la mayoría predominante en contraste con los demás medios planteados, tal como se muestra en la ilustración 2:

A su vez, se cuestionó acerca de la necesidad de contar con un código de ética que regule la profesión contable, en donde se planteó una escala del 1 al 5, donde 1 equivale a "No es necesario" y 5 a "Muy necesario", obteniendo como resultados que 98 de los encuestados, equivalente al 85\% del total señaló que la necesidad de un código de ética que regule la profesión contable es muy necesario, a diferencia de las demás opciones planteadas, tal como se puede apreciar en la ilustración 3:

\section{Ilustración 3. ¿Considera necesario un Código de ética que regule la Profesión Contable?}

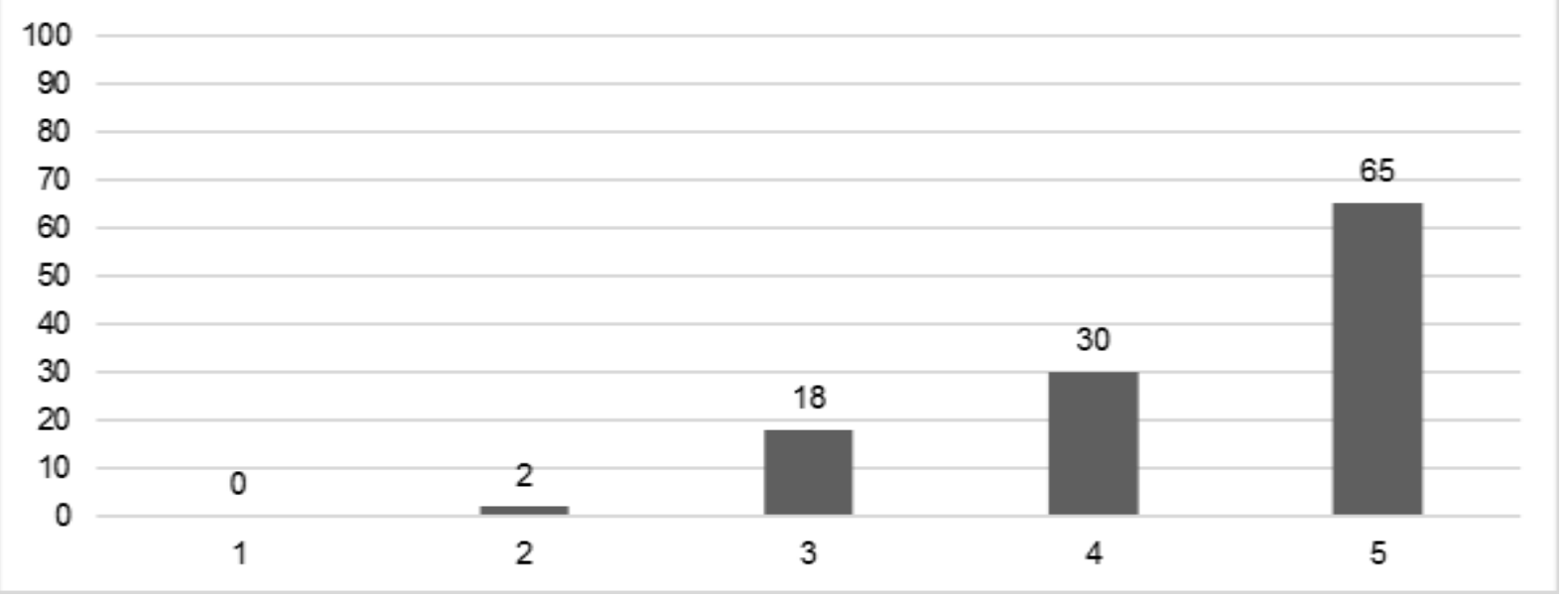

En relación al rol de las Universidades para brindar conocimientos sobre el Código de ética de la profesión

Contable, se cuestionó a los encuestados acerca de que tan necesario es que la ética profesional sea parte del mapa curricular de las Universidades, obteniendo como resultado que 65 de los encuestados, equivalente al $56.5 \%$ del total señaló que es "Muy importante" ya que la escala planteada fue de 1 al 5, donde 1 equivale a "No es necesario" y 5 a "Muy necesario", tal como lo muestra la ilustración 4: 


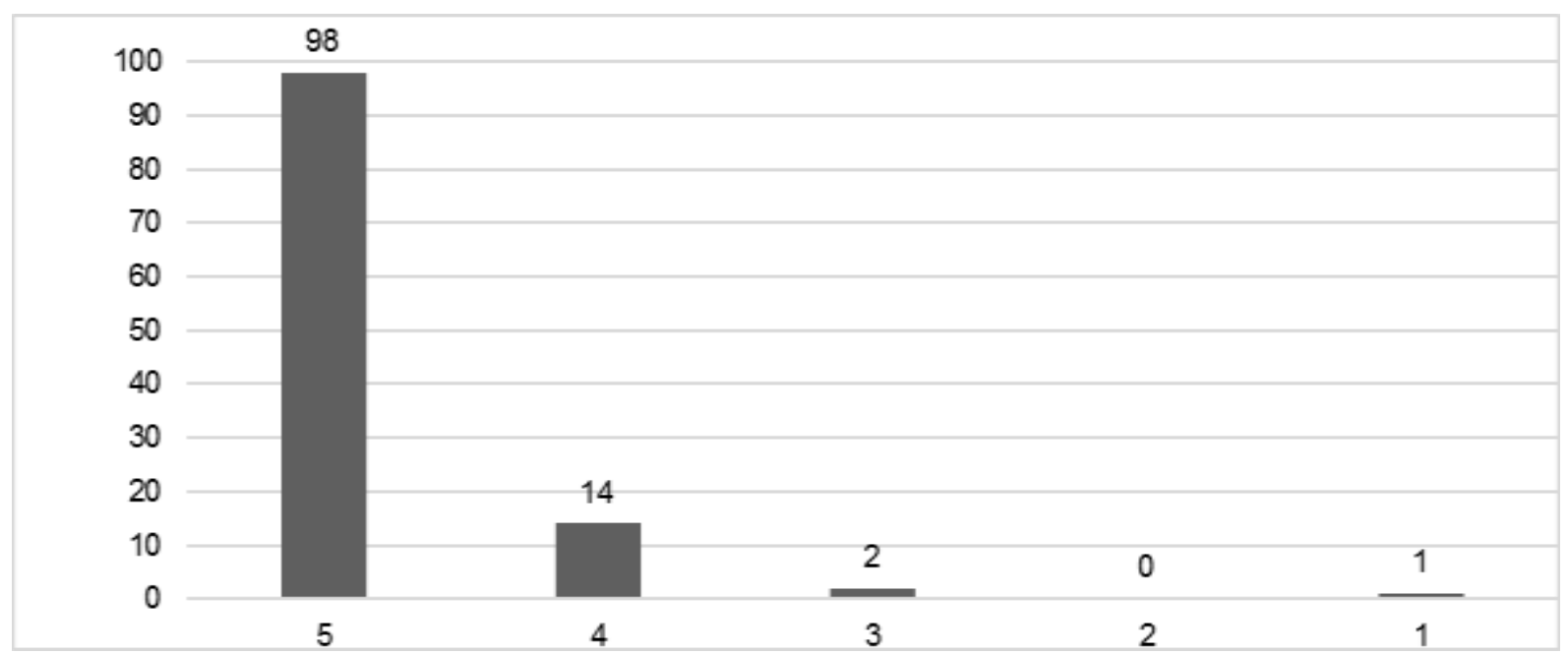

Ilustración 4. ¿Considera necesario que la ética profesional sea parte del mapa curricular de las Universidades?

Por otra parte, la encuesta se enfocó en que los profesionales contables calificaran en la escala Likert del 1 al 5 , el grado de frecuencia en la cual se han presentado dentro de su desempeño laboral las situaciones a continuación planteadas en la tabla 2, donde 1 equivale a "Nunca" y 5 equivale a "Siempre" tal como lo muestra la tabla 1:

\begin{tabular}{|c|c|}
\hline Respuesta de elemento & Valor \\
\hline Nunca & 1 \\
\hline Casi nunca & 2 \\
\hline A veces & 3 \\
\hline Casi siempre & 4 \\
\hline Siempre & 5 \\
\hline
\end{tabular}

Tabla 1. Valor de respuesta de elemento.

Como resultado, las situaciones planteadas con mayor frecuencia de preferencia hacia un nivel de escala fueron la situación 2 "He maquillado estados financieros" en la cual el $84 \%$ encuestados señaló que "nunca" han realizado esta práctica, por otra parte, la situación 3 "Mis actos son congruentes con mis palabras" en la cual el 49\% de los encuestados señaló que sus actos "siempre" han sido congruentes con sus palabras, de igual manera, en la situación 4 "Considero que lo que estoy haciendo en mi trabajo no es ético", el 76\% los encuestados señaló que "nunca", es decir, que en su trabajo siempre actúan de manera ética, a su vez, en la situación 6 "Considero que mi comportamiento es ético" la opción "siempre" fue seleccionada por el 68\% de los encuestados, tal como se puede observar en la tabla 2: 


\begin{tabular}{|l|c|c|c|c|c|c|}
\hline \multicolumn{1}{|c|}{ ESCALA } & $\mathbf{1}$ & $\mathbf{2}$ & $\mathbf{3}$ & $\mathbf{4}$ & $\mathbf{5}$ \\
\hline \multicolumn{1}{|c|}{ SITUACIÓN PLANTEADA } & \multicolumn{5}{|c|}{ FRECUENCIA } \\
\hline $\begin{array}{l}\text { 1.- Mi trabajo se ha visto afectado por } \\
\text { conflictos de interés. }\end{array}$ & $35 \%$ & $14 \%$ & $30 \%$ & $14 \%$ & $8 \%$ \\
\hline 2.- He "maquillado" estados financieros. & $84 \%$ & $2 \%$ & $7 \%$ & $4 \%$ & $0 \%$ \\
\hline $\begin{array}{l}\text { 3.- Mis actos son congruentes con mis } \\
\text { palabras. }\end{array}$ & $5 \%$ & $3 \%$ & $15 \%$ & $29 \%$ & $49 \%$ \\
\hline $\begin{array}{l}\text { 4.- Considero que lo que estoy haciendo en } \\
\text { mi trabajo no es ético. }\end{array}$ & $76 \%$ & $10 \%$ & $8 \%$ & $1 \%$ & $5 \%$ \\
\hline $\begin{array}{l}\text { 5.- Mis compañeros de trabajo se } \\
\text { comportan de manera legal y ética. }\end{array}$ & $3 \%$ & $5 \%$ & $28 \%$ & $25 \%$ & $38 \%$ \\
\hline $\begin{array}{l}\text { 6.- Considero que mi comportamiento es } \\
\text { ético. }\end{array}$ & $1 \%$ & $2 \%$ & $4 \%$ & $25 \%$ & $68 \%$ \\
\hline
\end{tabular}

Tabla 2. Frecuencia de situaciones planteadas.

Teniendo lo anterior en consideración, se puede deducir que las opciones con mayor frecuencia de preferencia hacia un nivel de escala fueron aquellas que depende del actuar ético personal, ya que en las situaciones 1 y 5 , hacían referencia a influencias externas del individuo, como lo conlleva los conflictos de interés y el comportamiento de los compañeros de trabajo.

\begin{tabular}{|c|c|}
\hline Respuesta de elemento & Valor \\
\hline No es importante & 1 \\
\hline Poco importante & 2 \\
\hline Neutral & 3 \\
\hline Importante & 4 \\
\hline Muy importante & 5 \\
\hline
\end{tabular}

Tabla 3. Valor de respuesta de elemento.

Para finalizar, la encuesta solicitó a los profesionales contables seleccionar el nivel de importancia que tiene cada principio del Código de ética profesional en su desempeño laboral, en la escala Likert del 1 al 5, donde 1 equivale a "No es importante" y 5 equivale a "Muy importante", tal como lo muestra la tabla 3 : 


\begin{tabular}{|l|c|c|c|c|c|}
\hline \multicolumn{1}{|c|}{ ESCALA } & $\mathbf{1}$ & $\mathbf{2}$ & $\mathbf{3}$ & $\mathbf{4}$ & $\mathbf{5}$ \\
\hline \multicolumn{1}{|c|}{ PRINCIPIO } & \multicolumn{6}{c|}{ FRECUENCIA } \\
\hline Integridad. & $0 \%$ & $0 \%$ & $2 \%$ & $12 \%$ & $86 \%$ \\
\hline Objetividad. & $0 \%$ & $0 \%$ & $2 \%$ & $14 \%$ & $84 \%$ \\
\hline Diligencia y competencia profesional. & $0 \%$ & $1 \%$ & $3 \%$ & $15 \%$ & $82 \%$ \\
\hline Confidencialidad. & $0 \%$ & $1 \%$ & $1 \%$ & $10 \%$ & $89 \%$ \\
\hline Comportamiento profesional. & $0 \%$ & $1 \%$ & $0 \%$ & $10 \%$ & $89 \%$ \\
\hline
\end{tabular}

Tabla 4. Frecuencia de principios del Código de ética del Profesional Contable.

Como se puede apreciar, los resultados se inclinaron en gran porcentaje hacia al nivel 5 de la escala planteada, el cual equivale a "Muy importante", por lo que, una media del $86 \%$ de los profesionales contables consideran muy importante, todos y cada uno de los principios básicos del código de ética de la profesión contable y los implementan en su desempeño laboral, tal como se puede observar en la tabla 4:

\section{Discusión}

Para desempeñarse al más alto nivel de profesionalismo se debe tener siempre en consideración los principios fundamentales, que de acuerdo al Código de Ética Profesional emitido por el Instituto Mexicano de Contadores Públicos (2015, p.14) se conforman de 5 principios, siendo estos los siguientes:

Integridad: "Ser leal, veraz y honrado en todas las relaciones profesionales y de negocios".

Objetividad: "Evitar prejuicios, conflictos de interés o influencia indebida de terceros que afecten el juicio profesional o de negocios".

Diligencia y competencia profesional:

Mantener el conocimiento profesional y las habilidades al nivel necesario que aseguren que el cliente o la entidad para la que se trabaja reciben servicios profesionales competentes basados en los últimos avances de la práctica, la legislación y las técnicas, y actuar con diligencia y de conformidad con las normas técnicas y profesionales aplicables.

\section{Confidencialidad:}

Respetar la confidencialidad de la información obtenida como resultado de relaciones profesionales o de negocios y, por lo tanto, no revelar dicha información a terceros sin la autorización apropiada y específica, a 
menos que haya un derecho u obligación legal o profesional de hacerlo, ni usar la información para provecho personal o de terceros.

Comportamiento profesional: "Cumplir con las leyes y reglamentos relevantes, y evitar cualquier acción que desacredite a la profesión".

Seguir los lineamientos éticos y normativos es algo que todos los que ejercen esta profesión deben de hacer, pero en algunos casos, esto va más allá de las normas y leyes que rigen la contabilidad, se trata más bien de un trabajo personal que implica qué tanto nivel de moralidad y profesionalismo se tiene individualmente.

Para los Profesionales contables de la ciudad de Villahermosa, estado de Tabasco, México, la ética es de vital importancia en su actuar profesional, por lo que, en su desempeño laboral, evitan realizar prácticas que perjudiquen a la profesión y con ello a terceros, ya que, como se mencionó anteriormente, la información financiera es consultada por diversos usuarios, y de igual manera, las malas prácticas perjudican a la empresa, trayendo consigo consecuencias perjudiciales para el contribuyente, esto como resultado de discrepancia o inconsistencias en la información presentada ante las autoridades competentes.

A su vez, la percepción que tienen los profesionales de la ética es como un factor imprescindible y relevante, es decir, debe estar presente en cada una de las actividades que se realizan para garantizar la calidad de la información y con ella la adecuada toma de decisiones, es importante mencionar que la ética no se debe limitar a la elaboración de informes financieros, también debe estar presente en toda relación laboral ya que de esta manera se mantiene un sano ambiente de trabajo.

Respecto la ética profesional como parte de los programas curriculares, es indispensable que esta esté presente de manera general, para que, desde antes de comenzar a laborar, los futuros LCP estén correctamente informados acerca de las buenas prácticas y de aquellas situaciones que debe evitar, así como saber manejar correctamente las problemáticas que se presenten, evitando que éstos sean blanco fácil para los problemas de conflictos de interés, y manipulación.

Es necesario mencionar que en la presente investigación se observó que los participantes señalaron que en su actuar profesional personal ponen en práctica la ética, en contraste, estos de igual manera, señalaron que hay una falta de ética respecto a influencias externas del individuo, como lo conlleva los conflictos de interés y el comportamiento de los compañeros de trabajo. 


\section{Conclusión}

Es de vital importancia que la información financiera sea confiable y veraz, ya que garantiza trabajos de calidad y, una clara y precisa situación financiera de la empresa, lo que permite tomar decisiones acertadas acerca del futuro y desempeño de la misma, de igual manera, el ético desempeño del profesional en Contaduría Pública evita irregularidades en la presentación de la información económico-contable ante las autoridades correspondientes.

Es por ello que la ética es de suma importancia para el Profesional Contable, ya que garantiza el actuar honesto, y con ello la confiabilidad de la información financiera plasmada en los informes contables, los estudiantes y profesionales contables de la Ciudad de Villahermosa tienen en consideración que actuar de manera ética es indispensable para ser considerado un verdadero profesional.

La investigación realizada demuestra a su vez, que es indispensable ampliar la población de encuestados para amplificar la presente investigación respecto a la percepción que tienen los Profesionales Contables acerca de la importancia de la ética, ya que de esta manera se pueden generar nuevas perspectivas y variantes.

\section{Bibliografía}

Alva, J. (2018). Contabilidad, paradigma de los conjuntos: Etimología, historia y perspectivas futuras. Alternativa financiera, 9(1), 1-17.

Colmenares, L. Da Costa, M y Cegarra, M. (2014). Valores éticos de los estudiantes de contaduría pública del NURR - Trujillo. Educere, 18(61), 547-556.

Cifuentes, L. Endara, I. Reyes, P., y Rivas, L. (2018). Manejo Contable en la empresa. Dominio de las Ciencias, 4(1), 178-187.

Elizalde, L. (2019). Los estados financieros y las políticas contables.593 Digital Publisher CEIT, 4(5-1), 217226.

Fernández, S. Diaz, L. Rodríguez, W. y Martínez, L. (2019). Influencia de la tecnología e información para el rendimiento de las Mipymes colombianas. Actualidad Contable Faces, 22(38), 25-45.

García, R. (2020). Formación secretarios en la práctica de valores éticos. Revista Sinapsis, 1(16). 
Gómez, C. Aristizabal, C. y Fuentes, D. (2017). Importancia de la Información Financiera para el ejercicio de la Gerencia. Desarrollo Gerencial, 9(2), 88-101.

Hernández, S. Fernández, C. y Baptista, P. (2014). Metodología de la investigación. 6a ed. México: McGRAWHILL.

Instituto Mexicano de Contadores Públicos. (2015). Código de Ética Profesional. 10a Ed. México: CINIF.

Instituto Mexicano de Contadores Públicos. (2018). Normas de Información Financiera. 13a Ed. México: CINIF.

Ostengo, H. (2015). El Sistema de Información Contable. (p.10). $2^{\mathrm{a}}$ ed. Argentina: Osmar D. Buyatti.

Santos, C. (2018). Una mirada a la historia de la Contabilidad. Revista Cubana De Finanzas Y Precios, 2(1), $139-155$.

Universidad Naval. (2016). Metodología de Investigación. 